\title{
Validation study of the Mini-Mental State Examination in a Malay-speaking elderly population in Malaysia
}

\begin{abstract}
Background/Aims: In view of the differing sensitivity and specificity of the Mini-Mental State Examination (MMSE) in the non-English-speaking populations, we conducted the first validation study of the Malay version (M-MMSE) in Malaysia among 300 subjects (from the community and outpatient clinics). Methods: Three versions were used: M-MMSE-7(serial 7), M-MMSE-3 (serial 3) and M-MMSE-S (spell 'dunia'backwards). Dementia was assessed using the criteria of the Diagnostic and Statistical Manual of Mental Disorders IV . The optimal cutoff scores were obtained from the receiver operating characteristics curves. Results: Seventy-three patients (24.3\%) had dementia and 227 (75.7\%) were controls. Three hundred patients completed the M-MMSE-7, 160 the MMMSE-3 and 145 the M-MMSE-S. All 3 versions were valid and reliable in the diagnosis of dementia. The optimal cutoff scores varied with each version and gender. In the control group, significant gender differences were observed in the patients with the lowest educational status. Increasing educational levels significantly improved the M-MMSE performance in both genders. Conclusion: All 3 versions of the M-MMSE are valid and reliable as a screening tool for dementia in the Malaysian population, but at different cutoff scores.In those with the lowest educational background, gender adjusted cutoff scores should be applied.
\end{abstract}

Keyword: Mini-Mental State Examination; Malay; Dementia; Malaysia; Cutoff scores 